\title{
Autoimmune type 1 diabetes: resolved and unresolved issues
}

\author{
Abner Louis Notkins ${ }^{1}$ and Åke Lernmark ${ }^{2}$ \\ ${ }^{1}$ Experimental Medicine Section, Oral Infection and Immunity Branch, National Institute of Dental and Craniofacial Research, \\ NIH, Bethesda, Maryland, USA \\ ${ }^{2}$ Department of Medicine, Robert H. Williams Laboratory, University of Washington, Seattle, Washington, USA \\ Address correspondence to: Abner L. Notkins, National Institutes of Health, Building 30, Room 121, 30 Convent Drive, MSC 4322, \\ Bethesda, Maryland 20892-4322, USA. Phone: (301) 496-4535; Fax: (301) 402-4163; E-mail: anotkins@dir.nidcr.nih.gov.
}

J. Clin. Invest. 108:1247-1252 (2001). DOI:10.1172/JCI200114257.

Type 1 diabetes, also known as either juvenile diabetes (because of the early age of onset) or insulin-dependent diabetes mellitus (because of the clinical need for insulin), is now widely thought to be an organ-specific autoimmune disease. Here we wish to summarize some generally accepted facts about this disease and then give our perspectives on unresolved issues surrounding prediction, pathogenesis, and treatment. In particular, we will discuss markers of the disease, such as autoantibodies against pancreatic islet cell antigens.

\section{Prevalence and etiology of type 1 diabetes}

It is estimated that nearly a million people in the US are afflicted with this disease (1). The majority of the patients are diagnosed and classified with type 1 diabetes within the first two decades of life, but an increasing number of cases are being recognized in older individuals. The geographic incidence varies widely from $1.7 / 100,000$ per year in Japan to more than $35 / 100,000$ in Finland. In the US the lifetime prevalence approaches $0.4 \%$, but in high-incidence countries, such as Finland and Sweden, it may be as high as $1 \%$.

Type 1 diabetes is due to a deficiency of insulin as a result of destruction of the pancreatic $\beta$ cells. At the time of clinical symptoms, $60-80 \%$ of the $\beta$ cells are destroyed. Cells secreting glucagon, somatostatin, and pancreatic polypeptide are generally preserved but may be redistributed within the islets. Insulitis, an inflammatory infiltrate (Figure 1) containing large numbers of mononuclear cells and CD8 T cells, typically occurs around or within individual islets.

The cause of $\beta$ cell destruction remained an enigma for years, but two discoveries in the 1970s provided the basis for our current thinking about the disease. The first was a strong linkage of type 1 diabetes to the highly polymorphic HLA class II immune recognition molecules - DR and, later, DQ - located on chromosome $6(2,3)$. Over the years, extensive studies have revealed a large number of high- and low-risk HLA alleles (4-6). For example, whereas only $45 \%$ of the population in the US expresses DR 3 or DR $4,95 \%$ of those who develop type 1 diabetes express these haplotypes. A particularly strong association is found with the HLA haplotypes DQA $1 * 0301-\mathrm{B} 1 * 0302$, especially when combined with DQA $1 * 0501-\mathrm{B} 1 * 0201$. Other haplotypes show a strong negative association with type 1 diabetes. The DQA $1 * 0102-\mathrm{B} 1 * 0602$ haplotype occurs in over $20 \%$ of some populations, but less than $1 \%$ of children who develop type 1 diabetes express these alleles (7). HLA genotyping thus has become an important research tool for identifying subjects at risk of developing type 1 diabetes. Perhaps even more important, because of the known role of HLA molecules in antigen presentation, the HLA linkage and association supported the hypothesis that type 1 diabetes has an autoimmune component.

The second discovery, providing direct evidence for autoimmunity, came by incubating sera from type 1 diabetic patients with frozen tissue sections of normal blood group 0 pancreas $(8,9)$. Sera from type 1 diabetic patients with polyendocrine disease were found, by immunofluorescence, to stain pancreatic islets. These antibodies, which came to be known as islet cell anti-

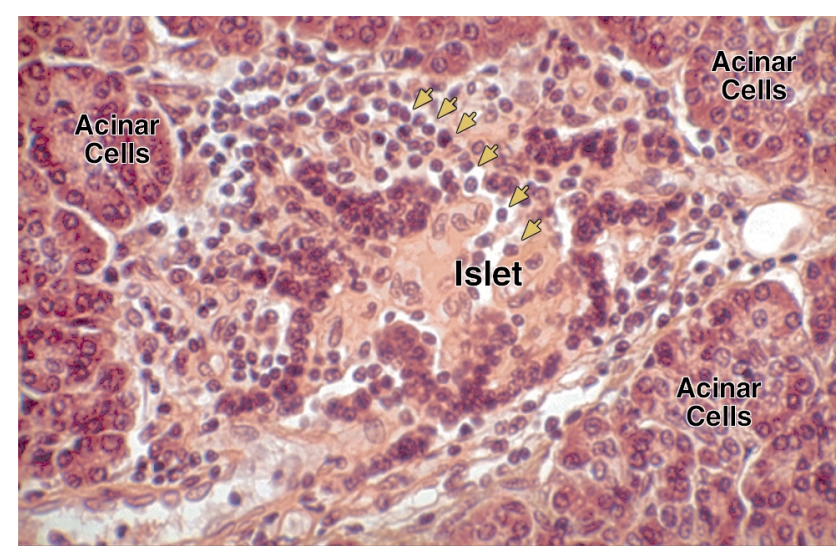

Figure 1

Inflammatory infiltrate of mononuclear cells in an islet from a 2-yearold patient with type 1 diabetes of short duration. Mononuclear cells in and around islets are shown by yellow arrows. This patient was reported by Willy Gepts in his original contribution on insulitis in 1965 (45). The photomicrograph comes from the collection of W. Gepts and was kindly provided by Danny Pipeleers. 
Table 1

Major autoantigens in type 1 diabetes

\begin{tabular}{lccc}
\hline & GAD65 & IA-2 & Insulin \\
Amino acid length & 585 & 979 & 51 \\
Molecular weight (Da) & 65,000 & 106,000 & 6,000 \\
Chromosome & $10 \mathrm{p} 11$ & $2 \mathrm{q} 35$ & $11 \mathrm{p} 15$ \\
Cell type in which & Neuroendocrine & Neuroendocrine & Pancreatic islet $\beta$ cells \\
expressed & pancreatic islet cells & pancreatic islet cells & \\
Intracellular location & Neuron-like small vesicles & Secretory vesicles & Secretory vesicles \\
Function & Converts glutamic acid to GABA: & Enzymatically inactive member & Ligand for the insulin receptor; \\
& inhibitory neurotransmitter & of PTP family & regulation of blood glucose \\
\end{tabular}

bodies (ICAs), have been widely used to study the clinical course and pathogenesis of type 1 diabetes, although the nature of the islet antigens involved remained unclear for a number of years.

\section{Identification of autoantigens}

In the 1980s and early 1990s the principal two autoantigens recognized by ICA were identified. The first was a new isoform of glutamic acid decarboxylase $(\operatorname{GAD} 65)(10,11)$ and the second was a protein tyrosine phosphatase-like molecule (IA-2) (12). The availability of these proteins in recombinant form allowed for the development of radioimmunoassays, which have now virtually replaced the ICA immunofluorescence test for measuring autoantibodies. A third antigen, insulin, also was identified in the 1980s (13). This antigen is not recognized in the ICA test, which uses unfixed frozen tissue sections from which insulin and c-peptide leach out during sample preparation.

There are two isoforms of GAD, one with a molecular weight of 65,000 (GAD65) and the other with a molecular weight of 67,000 (GAD67) $(14,15)$. GAD65 is involved in the conversion of glutamic acid to $\gamma$-aminobutyric acid (GABA), a major inhibitory neurotransmitter. The two isoforms are approximately $65 \%$ identical and are expressed not only in neurons, but also in pancreatic islet cells, where GAD65 predominates. The function of GAD65 in islet cells is not known. The GAD65 gene, which is found on chromosome $10 \mathrm{p} 11$, encodes a protein of 585 amino acids (Table 1). Autoantibodies in sera of type 1 diabetic patients are directed primarily to middle (amino acids 245-449) and C-terminal (amino acids 450-585) regions of the molecule (16). Some GAD65 autoantibodies in diabetes sera cross-react with GAD67, the product of an unlinked but homologous gene.

IA-2, also known as ICA512, is an unusual member of the transmembrane protein tyrosine phosphatase (PTP) family and is located on chromosome 2 q35 (16). It is atypical in that it lacks enzymatic activity because of a critical amino acid substitution at position 911 (Asp for $\mathrm{Ala}$ ) in the catalytic domain of the molecule. The molecular weight is 106,000 and the protein is 979 amino acids in length. The protein is expressed in neuroendocrine tissues and is found in both the $\alpha$ and the $\beta$ cells of the pancreatic islets. Immunofluorescence studies have localized IA- 2 to the secretory vesicles of both endocrine and neuronal cells, but its function is not known.
A closely related protein, and another autoantigen in type 1 diabetes, is IA-2 $\beta$, also known as phogrin (17). The intracellular domain of this 111,000 molecular weight protein is $74 \%$ identical to IA-2. IA- $2 \beta$, which is encoded on chromosome $7 q 36$, carries the same amino acid substitution in its catalytic domain, and it is also expressed in neuroendocrine tissues. IA- 2 and IA- $2 \beta$ belong to a highly conserved family of proteins with homologues in macaques, mice, rats, cows, zebra fish, Caenorhabditis elegans, and Drosophila (18). Autoantibodies to IA- 2 and IA- $2 \beta$ are directed exclusively to their intracellular domains (16). Most sera that recognize IA- $2 \beta$ also recognize IA- 2 , but not all sera that recognize IA-2 recognize IA- $2 \beta$. For this reason IA- 2 is the protein of choice for most immunoassays.

Insulin, the third autoantigen in type 1 diabetes (13), is a short protein of 51 amino acids encoded on chromosome 11p15. As with the GAD65- and IA-2-specific antibodies (16), autoantibodies in the sera of type 1 diabetic patients are directed primarily to conformational epitopes. In the case of insulin, these epitopes map to the B chain of human proinsulin or insulin (19). Unlike the GAD65 and IA-2 autoantibodies, insulin autoantibodies are not useful for confirming the classification of diabetes after insulin replacement therapy has begun, because patients develop antibodies to exogenous insulin.

Extensive studies have been carried out at international workshops to standardize the assays for autoantibodies to GAD65/IA-2/insulin (20,21). In the case of IA-2 and GAD65, radioligand binding assays have given precise and reproducible results. In the case of insulin, however, there has been considerable interlaboratory variation. A recently developed assay (22) may circumvent some of the problems with the older assay and may make it possible to measure all three autoantibodies reproducibly, using small volumes of serum. Autoantibodies to other antigens also have been reported (e.g., carboxypeptidase H, ICA69, GLUT-2, SOX-13, and $\beta$ cell sulfatides), but, because they are found at considerably lower frequency than autoantibodies to GAD65/IA-2/insulin, they have not been used in routine clinical studies.

\section{Autoantibodies as predictors of type 1 diabetes} Hundreds of studies have now been carried out in laboratories around the world to determine the relationship between autoantibodies to GAD/IA-2/insulin and 
type 1 diabetes $(16,23-27)$. Approximately $70-80 \%$ of newly diagnosed type 1 diabetes patients have autoantibodies to GAD65. Nearly the same number or slightly less have autoantibodies to IA-2. Overall, fewer patients appear to have insulin autoantibodies, but this is due to a pronounced age effect: children with newly diagnosed type 1 diabetes have a markedly higher frequency of autoantibodies to insulin than teenagers or young adults (23). Some patients carry autoantibodies to only one of the major autoantigens, but others may react to all three. In newly diagnosed subjects, up to $90 \%$ have autoantibodies to one or more of these antigens. The percent positivity depends on a variety of factors, including not only the age of the subjects, but also the duration of the disease and, in some cases, their ethnic origins. Some intrinsic variability is also seen in the assay, particularly at the limit of its range of detection. In general, GAD65 autoantibody positivity tends to be stable, whereas IA-2 autoantibodies tend to decrease with duration of disease, and insulin autoantibodies cannot be usefully measured after initiation of insulin therapy. Extensive analyses of these autoantibodies in normal controls suggest that about $1.0 \%$ have autoantibodies to IA-2, GAD65, or insulin.

Although initial interest focused on the presence of autoantibodies at the time of diagnosis and during follow-up of patients, it soon became apparent that these autoantibodies precede the development of diabetes by many months or years (see Leslie, this Perspective series, ref. 28). A large number of studies have now shown that the presence of autoantibodies in an otherwise healthy subject can be used as a predictive marker to identify subjects at high risk for type 1 diabetes (16, 23-26) (Figure 2). The highest risk is associated with subjects carrying autoantibodies to two or three of these autoantigens. For example, one study showed that the presence of both GAD65 and IA-2 autoantibodies carried nearly a 50\% risk of developing type 1 diabetes within 5 years and an even higher risk within 10 years (16). Other studies show that when subjects carry autoantibodies not only to IA-2 and GAD65, but also to insulin, the risk of developing diabetes within 5 years is increased to at least $70 \%$ (24). For the purpose of prediction, the presence of autoantibodies to two or more antigens is far more strongly associated with the risk of disease than is a high titer of autoantibody to any single antigen. Moreover, the combination of high-risk HLA genes with autoantibodies further increases positive prediction, and preliminary data suggest that autoantibodies appear earlier in children with high-risk genes $(29,30)$.

Populations are currently being screened for autoantibodies to GAD65, IA-2, and insulin to identify highrisk subjects for entry into therapeutic intervention trials. The value of this approach is obvious when one considers that the prevalence of individuals at risk of type 1 diabetes in the general US population is only 1 in 400 individuals. Therefore, to study the effect of a test drug on 100 subjects who will come down with type 1 diabetes, over 40,000 individuals would have to participate in the study. In contrast, with autoantibody markers, only 200 doubly autoantibody-positive sub- jects would be required, since almost $50 \%$ of these highrisk subjects will become diabetic within 5 years.

\section{Autoantibodies in adults}

Autoantibody screening is also being used to identify patients with type 1 diabetes in the older age group and in subjects initially classified as having type 2 diabetes. Diagnosis of diabetes follows internationally accepted thresholds of blood glucose. Classification of diabetes is based on clinical symptoms and treatment requirements rather than knowledge of the etiology and pathogenesis. This approach to classify a disease is unique to diabetes. It is therefore not surprising that screening of type 2 diabetes patients first by the ICA method and more recently by use of recombinant antigens has shown that $5-10 \%$ have autoantibodies to GAD65 and a somewhat lower percentage $(2-4 \%)$ to IA-2 (31-33). Very few older subjects have autoantibodies to insulin. Based on these findings, GAD65 appears to be the predominant autoantigen in the older age group with autoimmune diabetes. Taken together, these studies are showing that many patients who were thought to have type 2 diabetes either have a combination of type 1 and type 2 diabetes or have been misclassified and actually have type 1 diabetes. This type of diabetes has also been referred to as latent autoimmune diabetes in the adult (LADA), type 1.5 diabetes, and slowly progressive insulin-dependent diabetes mellitus (SPIDDM). Moreover, it should be noted that in some patients classified as having type 2 diabetes who nonetheless carry autoantibodies to GAD65, the fasting c-peptide and body mass index are low, and the likelihood of ultimately requiring insulin is remarkably high (34). Since there are nearly 16 million people in the US with type 2 diabetes, if only $5 \%$ have been misclassified, the number of patients with type 1 diabetes would be nearly double the current estimate.

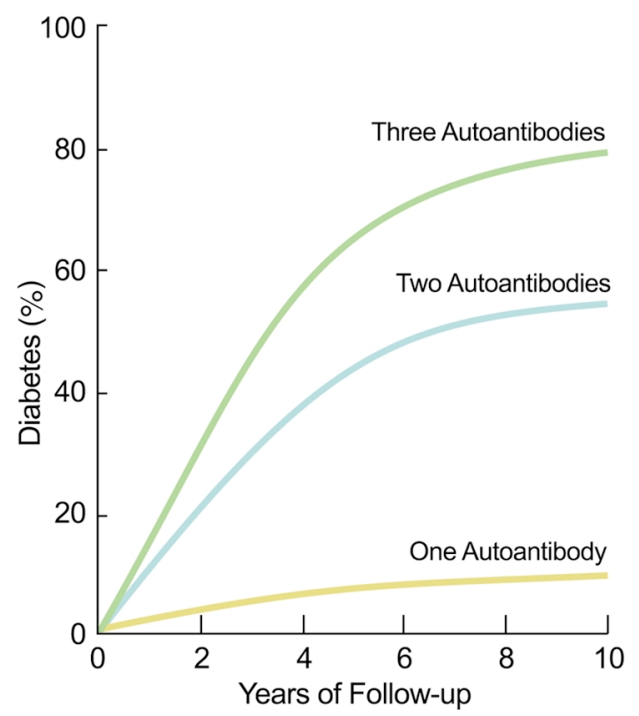

Figure 2

Diagrammatic representation of the effect of multiple autoantibodies (GAD65, IA-2, or insulin) on the risk of developing type 1 diabetes. Percent diabetes represents an approximation from several longitudinal studies on first-degree relatives of type 1 diabetes patients. 
Major unresolved issues

Triggers of type 1 diabetes. Both genetic and environmental factors have been implicated as triggers in the pathogenesis of type 1 diabetes. Of particular importance are the HLA genes that are involved in antigen presentation. While certain HLA haplotypes, such as DQA $1 * 0301-\mathrm{B} 1 * 0302$ and $\mathrm{A} 1{ }^{*} 0501-\mathrm{B} 1 * 0201$, are positively associated with type 1 diabetes, others, such as DQA $1 * 0102-\mathrm{B} 1 * 0602$, are negatively associated with type 1 diabetes (35). The positive association is far from absolute and, in fact, decreases in strength with increasing age of onset (35). Similarly, the negative association does not eliminate the possibility of developing type 1 diabetes but appears to slow the process and makes the development of the disease unlikely before age 30 . Although the binding of antigens to different HLA molecules has been extensively studied, why some HLA haplotypes are positively associated and others negatively associated with type 1 diabetes is not clear.

Complete genome scanning of sib pairs with diabetes has revealed two quantitative trait loci (36), one linked to the HLA class II loci on chromosome 6 and the other linked to the promoter polymorphism of the Insulin gene on chromosome 11. Multiple other loci that may contribute to type 1 diabetes risk have been reported, but often at low frequency, and such reports are not always confirmed. Genes that might affect immunological tolerance have figured prominently in the speculation about pathogenesis. However, the role of these multiple loci in the disease process has not been defined. It is still unclear to what extent they actually contribute to genetic risk beyond that already conferred by HLA class II, which has been estimated to account for nearly $50 \%$ of the genetic risk. Taken together with family studies, these findings support the argument that type 1 diabetes is a multifactorial disease and that there may be no specific diabetes gene, but only specific, "wrong" combinations of normal polymorphisms.

Environmental factors such as pathogens, toxins, drugs, and food components have long been thought to contribute to the pathogenesis of type 1 diabetes, with viruses being the leading environmental candidate $(37,38)$. Animal studies have shown that viruses can infect pancreatic $\beta$ cells and that the development of diabetes is dependent upon both the genetic background of the animal and the viral strain. Indeed, a single amino acid substitution within the virus can determine whether or not diabetes develops (39). In human twins, the development of type 1 diabetes in one twin is associated in less than half of cases with disease development in the identical co-twin, arguing that an environmental factor is required to trigger the disease. Of particular importance, susceptibility among identical twins is superimposed upon a diabetes-prone genetic background.

Case reports going back 100 years have shown a temporal relationship between certain viral infections and the development of type 1 diabetes, and seroepidemiologic studies provide additional support for this model $(27,37,38,40)$. Perhaps the strongest evidence for viral involvement in human type 1 diabetes comes from congenital rubella (41). Approximately $20 \%$ of children born with this infection ultimately develop type 1 diabetes. It is assumed that rubella either destroys a critical number of $\beta$ cells, or triggers an autoimmune response to $\beta$ cells, or both, in children with type 1 diabetes-susceptible HLA genes. Despite major efforts and numerous investigations of a large number of viruses in relation to diabetes onset, a viral cause for the vast majority of patients with type 1 diabetes has not been established. However, the demonstration that type 1 diabetes is a chronic autoimmune disease has changed the emphasis in the search for a viral agent from one that rapidly destroys $\beta$ cells to one that may establish a chronic infection or trigger an autoimmune response to $\beta$ cell antigens.

Pathogenesis of type 1 diabetes disease in bumans. Although there is extensive information on the pathogenesis of autoimmune diabetes in the NOD mouse and the BB rat, there is relatively little direct information on the pathogenesis of type 1 diabetes in humans. In fact, many of our ideas about the pathogenesis of human type 1 diabetes are the result of extrapolation from rodents. The NOD mouse has proved to be an excellent model for studies on autoimmunity, but there are considerable differences between the mouse model and the human disease, and direct information on etiology and pathogenesis at the human level is still needed.

The perceived hazards in performing pancreatic biopsies, which would be of great value in following the course of the disease over time, have greatly limited our knowledge. What is known about the pathogenesis of human type 1 diabetes comes largely from random autopsy specimens obtained from patients who died either near the time of diagnosis from acute metabolic diabetic ketoacidosis or late in the course of the disease. In both cases the findings probably represent a late stage of the disease many months or years after the initial triggering event. Morphological techniques show a marked decrease in $\beta$ cells over time, consistent with a chronic autoimmune inflammation, probably driven by macrophages and lymphocytes surrounding and infiltrating the islets. Because of the paucity of viable pancreatic islets, only limited information is available on the nature and function of the infiltrating cells. CD8 T cells seem to predominate, but neither the proportion of CD4 and CD8 cells nor the significance of other cell types in the infiltrate (e.g., natural killer cells, macrophages, dendritic cells) is known. Moreover, the proportion of CD4 or CD8 cells specific for GAD/IA-2/insulin is not well defined.

A variety of cell-mediated immune models have been proposed to explain the autoimmune destruction of $\beta$ cells, but none of these models have been documented at the human level. It is generally thought that $\beta$ cells expressing HLA class I antigens with peptides from one or more of the major autoantigens or from environmental triggers (e.g., viruses) might be recognized by and serve as targets for destruction by specific cytotoxic CD8 T cells. Surrogates of cell-mediated cytotoxicity using proliferation of peripheral blood $\mathrm{T}$ cells have shown that some patients with type 1 dia- 
betes recognize one or more of these major autoantigens, but often only a small fraction of patients respond, and it is sometimes difficult to quantitatively distinguish the response of patients from that of controls. This difficulty may be due to the fact that pathogenic $T$ cells are concentrated in the islets and are only sparsely represented in the peripheral blood. Assays for evaluating the cell-mediated response of patients and controls to diabetes-related autoantigens have also proved difficult to standardize. The whole area of islet pathogenesis would benefit enormously if noninvasive and quantitative techniques could be developed for estimating residual islet mass and the profile of inflammatory cells.

Of great importance, but still unresolved, is whether the infiltrating inflammatory cells themselves express cytokines that lead to cellular cytotoxicity or autoantibody formation. A cocktail of IL-1 $\beta$, INF- $\gamma$, and TNF- $\alpha$ is known to be toxic to human islet cells in vitro (42). ELISPOT or soluble HLA class II tetramer assays might be used to characterize the responses of individual peripheral or infiltrate-associated $\mathrm{T}$ cells and should help resolve the role of these cytokines. An alternative approach, although perhaps less convincing, is the measurement of immunoglobulin isotypes, which are thought to be surrogate markers for identifying Th1 and Th2 responses. These studies have shown that the autoantibodies to IA-2 and GAD65 are primarily of the IgG1 isotype, which argues in favor of a cytotoxic, Th1-type immune response in autoimmune type 1 diabetes (33).

Clinical trials. The discovery of autoantibodies and their value as predictors of type 1 diabetes is making it possible to identify subjects for clinical intervention trials long before their $\beta$ cell reserve is lost. The majority of these ongoing and proposed trials $(6,29,30)$ are designed to induce immunological tolerance or to suppress the immune response. These approaches are promising but face obstacles. The fact that there are three major autoantigens in type 1 diabetes makes it difficult to know whether one, two, or all three major autoantigens are needed to induce immunological tolerance. It is also uncertain whether there is a single triggering autoantigen and whether the development of the autoantibodies to secondary autoantigens results from epitope spread or a bystander response (see Wucherpfennig, this Perspective series, ref. 43). Indeed, despite innumerable studies, it is not absolutely clear whether autoimmunity in type 1 diabetes is the cause or the result of the disease process. It remains possible that a still-unrecognized combination of genes or environmental factors triggers the destruction of $\beta$ cells and that the autoimmune response is secondary to this process. Also of concern is the fact that $10-20 \%$ of newly diagnosed type 1 diabetes patients and an even higher percentage of African and Hispanic patients do not carry autoantibodies to any of the major autoantigens (7). These findings suggest either that the autoantibodies are present in very low titer or that other stillunidentified autoantigens are involved. Alternatively, a fraction of type 1 diabetes may not be autoimmune in nature (44). More precise information about the mech- anisms involved in the pathogenesis of human type 1 diabetes is needed for designing effective clinical trials.

\section{Summary}

Based on the presence of autoantibodies and a strong HLA linkage, type 1 diabetes is now classified as a chronic autoimmune disease. Many issues, however, remain unresolved. Although autoantibodies to GAD65, IA-2, and insulin are clearly markers for this disease, it is not known whether they contribute to pathogenesis or are simply the response to an existing underlying destructive process. Based on extensive studies in animal models, it is thought that it is the cellmediated immune response that is actually responsible for the destruction of $\beta$ cells. However, this has not been unequivocally established in humans because of the lack of a reliable assay for measuring cell-mediated immunity to $\beta$ cell antigens. What triggers the autoimmune response also is not known. The search for type 1 diabetes-specific genes so far has not been revealing, and environmental triggers, although widely viewed as important, have remained elusive. Despite enormous interest in the basis of the disease, type 1 diabetes pathogenesis remains understudied because of the difficulty and hazards in biopsying the pancreas.

Nevertheless, the studies on autoimmunity have provided clinically useful information. In particular, the demonstration of the presence of autoantibodies years before the onset of clinical symptoms has made it possible to identify individuals at high risk of developing type 1 diabetes and to initiate therapeutic intervention trials on relatively small numbers of subjects. Thus, to a very large degree, type 1 diabetes is a predictable disease. In addition, the demonstration of autoantibodies in $5-10 \%$ of individuals who were classified with type 2 diabetes suggests either that some of these individuals have a combination of type 1 and type 2 diabetes or that the number of patients with type 1 diabetes may be nearly twice as great as previously thought.

1. LaPorte, E.R., Matsushima, M., and Chang, Y.F. 1995. Prevalence and incidence of insulin-dependent diabetes. In Diabetes in America. 2nd edition. M.I. Harris, editor. NIH publication number 95-1468. Bethesda, Maryland, USA. 37-46.

2. Nerup, J., Platz, P., and Anderssen, O.O. 1974. HL-A antigens and diabetes mellitus. Lancet. 2:864-866.

3. Singal, D.P., and Blajchman, M.A. 1973. Histocompatibility (HL-A) antigens, lymphocytotoxic antibodies and tissue antibodies in patients with diabetes mellitus. Diabetes. 22:429-432.

4. She, J.X. 1996. Susceptibility to type 1 diabetes: HLA-DQ and DR revisited. Immunol. Today. 17:323-329.

5. Nepom, G.T., and Kwok, W.W. 1998. Molecular basis for HLA-DQ associations with IDDM. Diabetes. 47:1177-1184.

6. Schranz, D., and Lernmark, Å. 1998. Immunology in diabetes: an update. Diabetes Metab. Rev. 14:3-29.

7. Gottleib, P.A., and Eisenbarth, G.S. 1998. Diagnosis and treatment of pre-insulin dependent diabetes. Annu Rev. Med. 49:391-405.

8. Bottazzo, G.F., Florin-Christensen, A., and Doniach, D. 1974. Islet cell antibodies in diabetes mellitus with autoimmune polyendocrine deficiencies. Lancet. 2:1279-1283.

9. MacCuish, A.C., Barnes, E.W., Irvine, W.J., and Duncan, L.J.P. 1974. Antibodies to pancreatic islet-cells in insulin-dependent diabetics with coexistent autoimmune disease. Lancet. 2:1529-1531.

10. Baekkeskov, S., et al. 1982. Autoantibodies in newly diagnosed diabetic children immunoprecipitate human pancreatic islet cell proteins. Nature. 298: $167-169$.

11. Baekkeskov, S., et al. 1990. Identification of the $64 \mathrm{~K}$ autoantigen in insulin-dependent diabetes as the GABA-synthesizing enzyme glutamic acid decarboxylase. Nature. 347:151-156. 
12. Lan, M.S., Wasserfall, C., Maclaren, N.K., and Notkins, A.L. 1996. IA-2, a transmembrane protein of the protein tyrosine phosphatase family, is a major autoantigen in insulin-dependent diabetes mellitus. Proc. Natl. Acad. Sci. USA. 93:6367-6370.

13. Palmer, J.P. 1987. Insulin autoantibodies: their role in the pathogenesis of IDDM. Diabetes Metab. Rev. 3:1005-1015.

14. Bu, D.-F., et al. 1992. Two human glutamate decarboxylases, $65-\mathrm{kDa}$ GAD and 67-kDa GAD, are each encoded by a single gene. Proc. Natl. Acad. Sci. USA. 89:2115-2119.

15. Karlsen, A.E., et al. 1991. Cloning and primary structure of a human islet isoform of glutamic acid decarboxylase from chromosome 10. Proc. Natl. Acad. Sci. USA. 88:8337-8341.

16. Leslie, R.D., Atkinson, M.A., and Notkins, A.L. 1999. Autoantigens IA-2 and GAD in Type I (insulin-dependent) diabetes. Diabetologia. 42:3-14.

17. Lu, J., et al. 1996. Identification of a second transmembrane protein tyrosine phosphatase, IA-2beta, as an autoantigen in insulin-dependent diabetes mellitus: precursor of the 37-kDa tryptic fragment. Proc. Natl. Acad. Sci. USA. 93:2307-2311.

18. Cai, T., Krause, M.W., Odenwald, W.F., Toyama, R., and Notkins, A.L. 2001. The IA-2 gene family: homologs in Caenorhabditis elegans, Drosophila and zebrafish. Diabetologia. 44:81-88.

19. Potter, K.N., and Wilkin, T.J. 2000. The molecular specificity of insulin autoantibodies. Diabetes Metab. Res. Rev. 1:338-353.

20. Verge, C.F., et al. 1998. Combined use of autoantibodies (IA-2 autoantibody, GAD autoantibody, insulin autoantibody, cytoplasmic islet cell antibodies) in type 1 diabetes: combinatorial islet autoantibody workshop. Diabetes. 47:1857-1866.

21. Mire-Sluis, A.R., Das, R.G., and Lernmark, Å. 2000. The world health organization international collaborative study for islet cell antibodies. Diabetologia. 43:1282-1292.

22. Williams, A.J.K., Bingley, P.J., Bonifacio, E., Palmer, J.P., and Gale, E.A.M. 1997. A novel micro-assay for insulin autoantibodies. J. Autoimmun. 10:473-478

23. Hagopian, W.A., et al. 1995. Glutamate decarboxylase-, insulin- and islet cell-antibodies and HLA typing to detect diabetes in a general population-based study of Swedish children. J. Clin. Invest. 95:1505-1511.

24. Verge, C.F., et al. 1996. Prediction of type I diabetes in first-degree relatives using a combination of insulin, GAD, and ICA512bdc/IA-2 autoantibodies. Diabetes. 45:926-933.

25. Bingley, P.J., et al. 1997. Prediction of IDDM in the general population: strategies based on combinations of autoantibody markers. Diabetes. 46:1701-1710.

26. Kulmala, P., et al. 1998. Prediction of insulin-dependent diabetes mellitus in siblings of children with diabetes: a population-based study. J. Clin. Invest 101:327-336.

27. Hyöty, H., et al. 1995. A prospective study of the role of coxsackie B and other enterovirus infections in the pathogenesis of IDDM. Childhood diabetes in Finland (DiMe) study. Diabetes. 44:652-657.

28. Leslie, D., Lipsky, P., and Notkins, A.L. 2001. Autoantibodies as predic- tors of disease. J. Clin. Invest. In press.

29. Ilonen, J., Simell, O., Knip, M., and Akerblom, H.K. 1998. Screening for genetic IDDM risk and prevention trials in infancy. Diabetes Metab. Rev. 14:188-189.

30. Norris, J.M., et al. 1996. Lack of association between early exposure to cow's milk protein and beta-cell autoimmunity. Diabetes autoimmunity study in the young (DAISY). JAMA. 276:609-614.

31. Schranz, D.B., et al. 1998. A simple and rapid microSepharose assay for GAD65 and ICA512 autoantibodies in diabetes. J. Immunol. Methods. 213:87-97.

32. Turner, R., et al. 1997. UKPDS 25: autoantibodies to islet-cell cytoplasm and glutamic acid decarboxylase for prediction of insulin requirement in type 2 diabetes. UK Prospective Diabetes Study Group. Lancet. 350:1288-1293

33. Hawa, M.I., et al. 2000. Antibodies to IA-2 and GAD65 in type 1 and type 2 diabetes: isotype restriction and polyclonality. Diabetes Care. 23:228-233.

34. Littorin, B., et al. 1999. Antibodies (ICA and GADA) at diagnosis of diabetes predict the subsequent need for insulin. Diabetes Care. 22:409-412.

35. Graham, J., et al. 1999. Negative association between type 1 diabetes and HLA DQB1*0602- DQA1*0102 is attenuated with age at onset. Swedish Childhood Diabetes Study Group. Eur. J. Immunogenet. 26:117-127.

36. Todd, J.A. 1999. From genome to aetiology in a multifactorial disease, type 1 diabetes. Bioessays. 21:164-174.

37. Knip, M., and Akerblom, H.K. 1999. Environmental factors in the pathogenesis of type 1 diabetes mellitus. Exp. Clin. Endocrinol. Diabetes. 107:S93-S100.

38. Jun, H., and Yoon, J. 2001. The role of viruses in Type I diabetes: two distinct cellular and molecular pathogenic mechanisms of virus-induced diabetes in animals. Diabetologia. 44:271-285.

39. Jun, H.S., Kang, Y., Notkins, A.L., and Yoon, J.W. 1997. Gain or loss of diabetogenecity resulting from a single point mutation in recombinant encephalomyocarditis virus. J. Virol. 71:9782-9785.

40. Dahlquist, G., Ivarsson, S., Lindberg, B., and Forsgren, M. 1995. Maternal enteroviral infection during pregnancy as a risk factor for childhood IDDM. Diabetes. 44:408-413.

41. Forrest, J.M., Menser, M.A., and Burgess, J.A. 1971. High frequency of diabetes mellitus in young adults with congenital rubella. Lancet. 2:332-334

42. Mauricio, D., and Mandrup-Poulsen, T. 1998. Apoptosis and the pathogenesis of IDDM: a question of life and death. Diabetes. 47:1537-1543.

43. Wucherpfennig, K.W. 2001. Mechanisms for the induction of autoimmunity by infectious agents. J. Clin. Invest. 108:1097-1104.

44. Imagawa, A., Hanafusa, T., Miyagawa, J., and Matsuzawa, Y. 2000. A novel subtype of type 1 diabetes mellitus characterized by a rapid onset and an absence of diabetes-related antibodies. Osaka IDDM Study Group. N. Engl. J. Med. 342:301-307.

45. Gepts, W. 1965. Pathologic anatomy of the pancreas in juvenile diabetes mellitus. Diabetes. 14:619-633. 